diagnosis of an ocular variant of Stickler syndrome with minimal or no systemic manifestations. Am J Ophthalmol 2002; 134: 728-734.

S Yoshida, Y Yamaji, R Kuwahara, A Yoshida, T Hisatomi, A Ueno and $T$ Ishibashi

Department of Ophthalmology, Kyushu University Graduate School of Medical Sciences, Fukuoka 812-8582, Japan

Correspondence: S Yoshida,

Tel: + 8192642 5648;

Fax: + 81926425663

E-mail: yosida@med.kyushu-u.ac.jp

Eye (2006) 20, 743-745. doi:10.1038/sj.eye.6702001; published online 8 July 2005
Sir,

\section{Gyrate atrophy with bilateral full thickness macular hole}

Gyrate atrophy of the choroid and retina, a rare autosomal recessive inborn error of amino-acid metabolism, is caused by mutation in the gene encoding the ornithine amino transferase with its onset in early childhood. ${ }^{1}$ Ocular findings include axial myopia, posterior subcapsular cataract, and typical well-demarcated circumferential patches of chorioretinal atrophy with scalloped margins. ${ }^{1}$

Choroidal neovascularization, ${ }^{2}$ keratoconus, ${ }^{3}$ cystoid macular edema, and epiretinal membrane formation ${ }^{4}$ have also been reported. We report bilateral macular holes in a patient with gyrate atrophy, which to the best of our knowledge has been not described in the literature (MEDLINE Search).
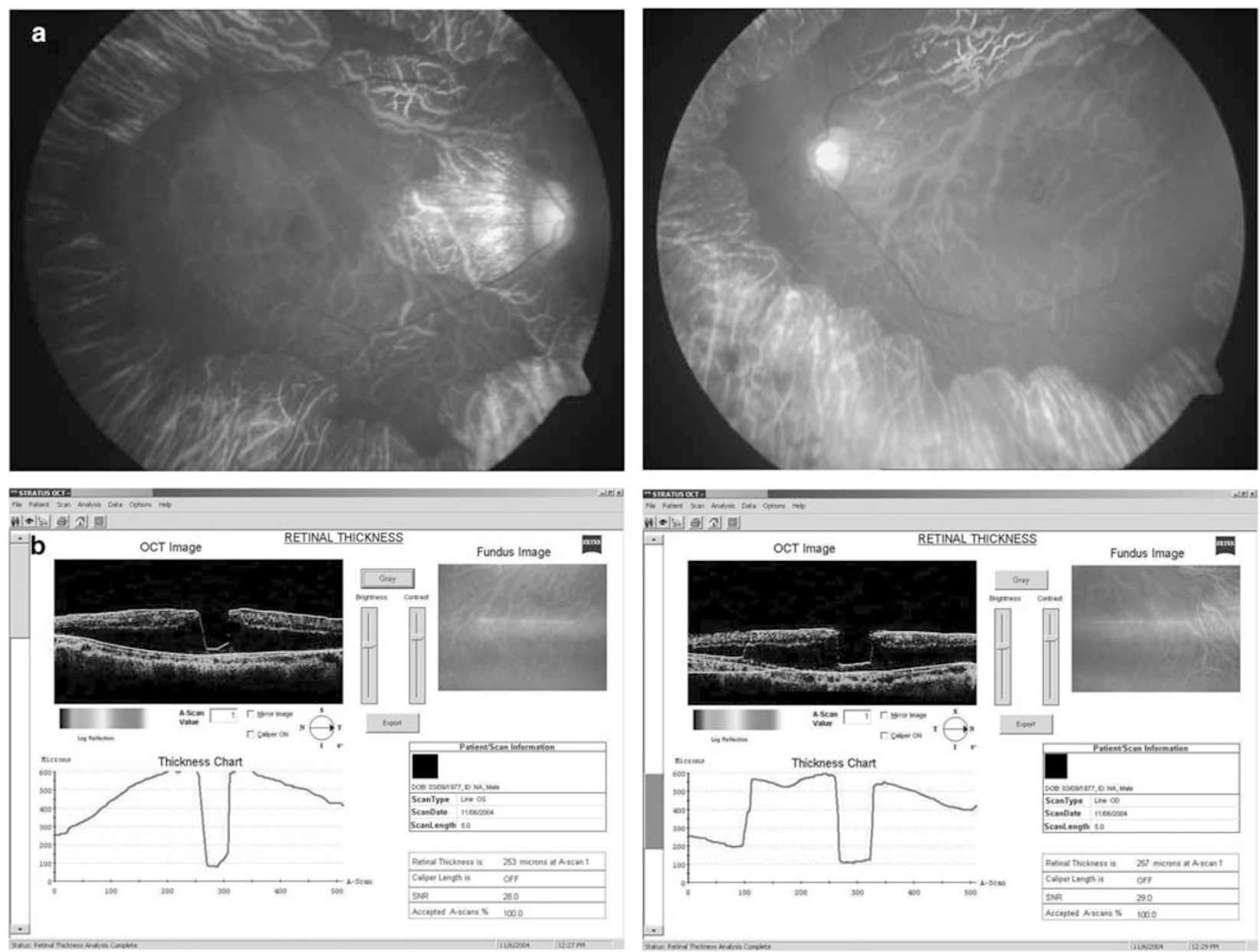

Figure 1 (a) Fundus photographs (top). Concentric chorioretinal atrophy with scalloped margins along with full thickness macular hole (FTMH) is seen in OD (top left) and OS (top right). (b) OCT Images (bottom). FTMH with surrounding neurosensory detachment can be seen in OD (bottom left) and OS (bottom right). 


\section{Case report}

A 28-year-male visited our vitreoretinal clinic with complaints of reduced peripheral vision and central scotoma in both eyes. His best-corrected visual acuity (BCVA) was 20/200 OD and 20/120 OS with a refractive error of $-3.5 \mathrm{D}$ sphere in both eyes. The patient had undergone bilateral phacoemulsification with posterior chamber intraocular lens implantation 3 years ago, when he was diagnosed with bilateral posterior subcapsular cataract with gyrate atrophy and hyperornithinemia (plasma ornithine $780 \mu \mathrm{mol} / \mathrm{l}$ ). The patient has been on an arginine-restricted diet since then. On examination, the fundus revealed bilateral full thickness macular holes (FTMH) with surrounding cuff of fluid along with typical concentric chorioretinal atrophy with scalloped margins (Figure 1a). Review of previous fundus photographs (with patient) taken prior to cataract surgery revealed similar bilateral FTMHs along with concentric chorioretinal atrophy (Figure 2a). FTMH was confirmed in both the eyes by optical coherence tomography (Figure 1b).

Both dynamic (Goldman) and automated static (Humphrey) perimetry found severe constriction of visual field in both eyes. Flash electroretinography revealed extinguished responses in both eyes. Comparison of recent photographs with earlier ones (3-years-old) showed some progression of gyrate atrophy (Figure 2a and b). Fresh plasma ornithine levels were found to be $370 \mu \mathrm{mol} / 1$.

The patient was counseled regarding argininerestricted diet, poor visual prognosis, genetic analysis, and also for vitreous surgery for FTMH, which he declined. Hence, he was prescribed pyridoxine phosphate (vitamin $\mathrm{B}_{6}$ ) $300 \mathrm{mg}$ daily and followed up. His BCVA and fundus photographs were unchanged at 4, 6 and 12 weeks follow-up while plasma ornithine levels decreased to $214 \mu \mathrm{mol} / 1$ at 12 weeks.

\section{Comments}

In gyrate atrophy, the affected individuals develop axial myopia in early childhood and most have impaired peripheral and night vision by age of 10 years. ${ }^{1}$ Pathological myopia with progressive thinning and stretching of posterior pole, thinning of choroid and ganglion cell layer, and consequent cystic degeneration, and atrophy of macula may possibly lead to macular hole formation. ${ }^{5}$ Posterior vitreous detachment and vitreous degeneration occur at a younger age in high myopes and resulting anteriorposterior traction can cause abrupt dehiscence of the macular tissue. ${ }^{5}$ Cataract surgery can explain the formation of macular holes by forward vitreous
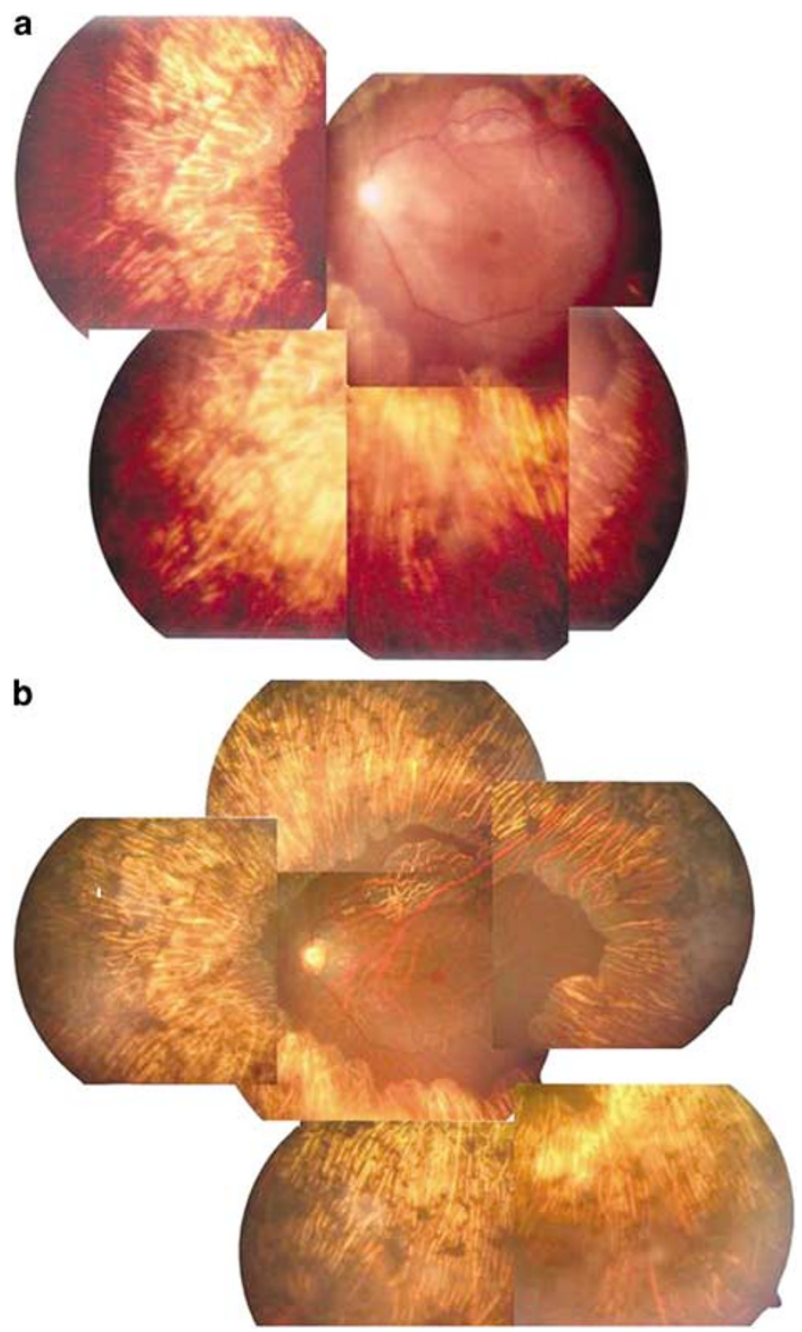

Figure 2 Comparisons of Montages showing the progression of chorioretinal atrophy. (a) Montage constructed from 3-year-old photographs. Macular hole is visible along with chorioretinal atrophy with scalloped margins. (b) Montage constructed from recent pictures. Progression of chorioretinal atrophy is seen.

displacement and cystoid macular edema, but existence of macular holes prior to cataract surgery precludes this possibility. Idiopathic FTMH most commonly occurs in sixth and seventh decades with the mean age reported in most series ranging from 66.33 to 69.5 years. ${ }^{5-7}$ The development of idiopathic FTMH in those below 30 years of age is rare.

We conclude that occurrence of a macular hole in a patient with gyrate atrophy is a rare event which leads to double jeopardy as it adds to the visual handicap of affected patients, whose peripheral visual fields are already severely constricted. This case could represent a real or chance association. Further investigations on similar cases, if found, can help elucidate the relationship between these two unusual findings. 


\section{References}

1 Potter MJ, Berson EL. Diagnosis and treatment of gyrate atrophy. Int Ophthalmol Clin 1993; 33(2): 229-236.

2 Marano F, Deutman AF, Pinckers AJ, Aandekerk AL. Gyrate atrophy and choroidal neovascularization. Arch Ophthalmol 1996; 114(10): 1295

3 Chen CJ, Furr P. Bilateral keratoconus in a patient with gyrate atrophy and hyperornithinemia. Am J Ophthalmol 1983; 95(5): 705-706.

4 Feldman RB, Mayo SS, Robertson DM, Jones JD, Rostvold JA. Epiretinal membranes and cystoid macular edema in gyrate atrophy of the choroid and retina. Retina 1989; 9(2): 139-142.

5 Judson PH, Yannuzzi LA, Macular Hole, Ryan SJ (eds). Retina. Mosby, Inc.: Singapore, 2001, pp 1196-1197.

6 Banker AS, Freeman WR, Azen SP, Lai MY, The Vitrectomy for Macular Hole Study Group. A multicentred clinical study of serum as adjuvant therapy for surgical treatment for macular holes. Arch Ophthalmol 1999; 117: 1499-1502.

7 Ezra E, Gregor ZJ, Moorfields Macular Hole Study Group Report No. 1. Surgery for idiopathic full-thickness macular hole. Arch Ophthalmol 2004; 122: 224-236.

YR Sharma, DV Singh, RV Azad and N Pal

Vitreoretina and Trauma Service, Dr Rajendra

Prasad Centre for Ophthalmic Sciences,

All India Institute of Medical Sciences,

Ansari Nagar, New Delhi 110029, India

Correspondence: DV Singh,

Tel: + 9111 26593193;

Fax: + 911126588919.

E-mail: deependravsingh@hotmail.com

Eye (2006) 20, 745-747. doi:10.1038/sj.eye.6702009; published online 8 July 2006

Sir,

Acute bilateral simultaneous PION after ectopic pregnancy-related haemorrhage

Acute bilateral simultaneous posterior ischaemic optic neuropathy (PION) is a rare visual complication of ectopic pregnancy-related haemorrhage. Only one other patient with acute visual loss related to haemorrhage from an ectopic pregnancy has been described.

\section{Case report}

This healthy 37-year-old gravida 4 para 3 woman presented with acute bilateral simultaneous severe visual loss 1 day after her exploratory laparotomy, left salpingectomy, bilateral tubal ligation, and total abdominal hysterectomy for her ruptured cornual ectopic pregnancy. Prior to her surgery she had 20/20 vision. Intra-operative haematocrit was 19.3\% (38-46\% normal), intraoperative blood pressure readings ranged from $110 / 50$ to $155 / 82 \mathrm{mmHg}$, and heart rate ranged from 58 to 110 beats $/ \mathrm{min}$. She received a total of $6100 \mathrm{ml}$ of crystalloid solution and her total estimated blood loss was $4000 \mathrm{ml}$. Postoperative haematocrit was $25.2 \%$ (38-46\% normal) at $1 \mathrm{~h}, 18.2 \%$ (38-46\% normal) at $6 \mathrm{~h}$, and $15.6 \%$ (38-46\% normal) at $12 \mathrm{~h}$. Postoperative blood pressure readings ranged from $100 / 50$ to $132 / 62 \mathrm{mmHg}$ On postoperative day one, she developed acute bilateral simultaneous visual loss associated with no headache or ocular pain. Her vision was NLP OU. Pupils were both $8 \mathrm{~mm}$ and nonreactive and round to light. Intraocular pressures were $17 \mathrm{mmHg}$ OD and $18 \mathrm{mmHg}$ OS.

Extraocular motility was normal. Both optic discs appeared normal without any evidence of edema. Head CT scan was normal and MRI of the brain and orbits with gadolinium 3 days later did not reveal any infarcts or optic nerve pathology. CSF analysis was also normal.

After 1 month, her visual acuity improved to 20/200 OD and 20/40 OS. Humphrey central 24-2 visual field testing revealed that she had generalized depression OD and peripheral constriction OS. She had a right relative afferent pupillary defect and a red-green colour defect. Extraocular motility was normal. Diffuse disc pallor was greater in the right optic disc than in the left. P100 latencies were increased at $122 \mathrm{~ms}$ OD and $117 \mathrm{~ms}$ OS (97-115 ms normal); P100 amplitudes were decreased at $1.1 \mu \mathrm{V}$ OD and $1.7 \mu \mathrm{V}$ OS (3-15 $\mu \mathrm{V}$ normal). The electroretinogram (ERG) was normal.

\section{Comment}

Visual loss is an uncommon but well-known complication of severe hypotension and anemia, most often occurring in the perioperative period. ${ }^{2}$ Decreased blood supply to pial arteries derived from the ophthalmic artery leads to ischaemia of the posterior optic nerve. ${ }^{3}$ The pathogenesis of ischaemic optic neuropathy is unclear, but is believed to be related to optic nerve hypoxia or ischaemia in severe anaemia, with or without arterial hypotension. ${ }^{4}$ Hayreh $^{4}$ noted that visual loss was not necessarily prevented in his patients who received immediate and adequate blood transfusion. He hypothesized that release of angiotensin, epinephrine, and vasopressin secondary to increased sympathetic activation could have caused vasoconstriction of the posterior optic nerve circulation resulting in posterior optic nerve ischaemia.

The diagnostic criteria for PION in this report are based upon those put forth by Buono et $a l^{5}$ which includes: (1) an acute decrease in visual acuity, visual field, or both; (2) an ipsilateral relative afferent pupillary 